\title{
Worldsheet instantons and $(0,2)$ linear models
}

\author{
Marco Bertolini $^{a, b}$ and M. Ronen Plesser ${ }^{a}$ \\ ${ }^{a}$ Center for Geometry and Theoretical Physics, \\ Box 90318, Duke University, Durham, NC 27708-0318, U.S.A. \\ ${ }^{b}$ Kavli Institute for Theoretical Physics, \\ University of California, Santa Barbara, CA 93106-4030, U.S.A. \\ E-mail: mb266@phy.duke.edu, plesser@cgtp.duke.edu
}

ABSTRACT: We study the stability of heterotic compactifications described by $(0,2)$ gauged linear sigma models with respect to worldsheet instanton corrections to the space-time superpotential following the work of Beasley and Witten [1]. We show that generic models elude the vanishing theorem proved there, and may not determine supersymmetric heterotic vacua. We then construct a subclass of linear models for which a vanishing theorem holds, generating an extensive list of consistent heterotic backgrounds.

KEYWORDS: Superstrings and Heterotic Strings, Superstring Vacua

ARXIV EPRINT: 1410.4541 


\section{Contents}

1 Introduction 1

2 The linear model $\quad 3$

3 The argument $\quad 6$

$\begin{array}{lll}3.1 & \text { The quintic } & 7\end{array}$

3.2 A counter-example 8

4 The vanishing theorem $\quad 9$

$\begin{array}{ll}4.1 & O \text { model gauge instanton moduli space } \\ & 10\end{array}$

$\begin{array}{lll}4.2 \text { A classical symmetry } & 10\end{array}$

5 Outlook 13

$\begin{array}{lr}\text { A Linear model conventions } & \mathbf{1 3}\end{array}$

$\begin{array}{lll}\text { A.1 } & (0,2) \text { superspace } & 13\end{array}$

$\begin{array}{ll}\text { A.2 Field content } & 14\end{array}$

$\begin{array}{lll}\text { A.3 The action } & 15\end{array}$

B The half-twist $\quad 16$

\section{Introduction}

A natural starting point for exploring the moduli space of $(0,2)$ heterotic compactifications is the study of the geometry of holomorphic vector bundles $\mathcal{V}$ over Calabi-Yau (CY) manifolds $M$. Under suitable conditions such bundles determine, to all orders in $\alpha^{\prime}$, a supersymmetric heterotic vacuum. For a long time it has been known [2] that worldsheet instantons wrapping rational curves in $M$ in principle generate a potential which destabilizes the vacuum. In rather special cases, such as models with $(2,2)$ supersymmetry [3] or some specially fine-tuned $(0,2)$ models $[4,5]$, the correction terms vanish for each instanton separately, but this is not true in more generic models [6-8].

In this context, heterotic compactifications obtained as gauged linear sigma models (GLSMs) [9] have received special attention, as they are believed to be stable under worldsheet instantons, even in the generic case in which the contributions of individual instantons do not vanish. This claim is then a nontrivial vanishing theorem about the total contribution from each instanton class. This was first proposed in [10] and further studied in [1]. ${ }^{1}$ This work suggests that in these models the corrections vanish even when the contributions of individual instantons do not. If true, this would guarantee the existence of a vast playground for tackling issues of $(0,2)$ moduli spaces.

\footnotetext{
${ }^{1}$ In [11] an argument along rather different lines was pursued.
} 
Essentially, these arguments rely on the fact that in heterotic vacua determined by $(0,2)$ theories the space-time superpotential for gauge singlets can be determined by a correlator $\mathcal{C}$ computed in a (half-) twisted version of the model. Unlike the twisted versions of $(2,2)$ theories, this is not a topological field theory, but the simplifications associated to the existence of a nilpotent scalar charge, such as the decoupling of exact operators from the correlators of closed operators, carry over to this case and show that $\mathcal{C}$ depends holomorphically on the relevant worldsheet couplings.

This holomorphy together with compactness arguments can be used to show that the correlator vanishes identically. The argument of [10] used the fact that the parameter space of the GLSM is compact (or has a natural compactification). $\mathcal{C}$ was shown to be a global section of a holomorphic bundle of negative curvature. If nonzero this must exhibit poles, which in this theory arise from the finite-energy configurations with very large field values which occur at special loci in the parameter space. At these loci the model is indeed singular, but the large-field region can be studied semiclassically to demonstrate that these configurations do not lead to any singularities in $\mathcal{C}$. The absence of poles shows that this vanishes identically. This was pursued explicitly in a simple example, but the argument did not appear to rely on details of this example so seemed likely to generalize.

In turn, the argument of [1] relied on the compactness of an appropriate moduli space of instantons. More precisely, these authors used the fact that the contribution to $\mathcal{C}$ at any fixed instanton number can be related to a calculation in a model in which many of the worldsheet couplings vanish. In this model, the moduli space of instantons is compact, and a zero-mode counting argument shows that the contribution to $\mathcal{C}$ vanishes. Here too, detailed calculations were done in simple examples but the argument seemed very robust and likely to hold in general.

In this note, we follow up on this work with a systematic study of the conditions under which the vanishing theorem of [1] applies. We find that in a generic gauged linear sigma model the argument that the moduli space of instantons is compact fails, reviving the question of whether these models are in fact destabilized by worldsheet instantons. We do not resolve this question. We are, however, able to construct an extensive class of models for which the argument holds - a sizable playground, if not as extensive as had been hoped.

The rest of the paper is organized as follows. In section 2 we review the construction of $(0,2)$ linear models relevant for our analysis. In section 3 we show that there exist models for which the vanishing of the space-time superpotential for gauge singlets is not guaranteed and we present an example in detail. In section 4 we prove a vanishing theorem for a particular subclass of $(0,2)$ linear models. In section 5 we end with some implications of this work and future directions.

\section{Acknowledgments}

We would like to thank Nick Addington, Paul Aspinwall, Chris Beasley, Ilarion Melnikov, Eric Sharpe and Edward Witten for useful conversations. MB would like to particularly thank Ilarion Melnikov for pointing out the problem and for countless discussions. This 
work is supported in part by the NSF Grant PHY-1217109 (MB and MRP) and NSF Grant PHY11-25915 (MB). We thank the organizers of the workshop on Heterotic Strings and $(0,2)$ QFT at Texas A\&M University in May, 2014 where this project began. MRP thanks the particle physics group at Tel Aviv University, and especially Yaron Oz, for their gracious hospitality while pursuing this work.

\section{The linear model}

Our tool for investigating the issue of instanton corrections in this note is the $(0,2)$ gauged linear sigma model. For a suitably constructed bundle $\mathcal{V}$ on a CY space $M$ presented as a complete intersection $H_{A}=0$ in a Fano toric variety $V,{ }^{2}$ the IR worldsheet dynamics is expected to be the same as that of an Abelian gauge theory with $(0,2)$ supersymmetry. In this section we are going to review the construction of the $(0,2)$ linear model [9] in order to establish notation and define the class of models we consider. More details can be found in appendix A.

The linear models we consider are gauge theories with gauge group $\mathrm{U}(1)^{R}$, along with $m$ neutral chiral supermultiplets we call $\Sigma_{\mu}=\left(\sigma_{\mu}, \lambda_{\mu,+}\right)$. We couple these to a collection of charged supermultiplets determined by the geometric data:

$$
\begin{array}{ccccccc}
\text { fields } & P^{\alpha} & \Phi^{i} & \Gamma^{I} & \Lambda^{A} & S & \Xi \\
\mathrm{U}(1)^{a} & -m_{\alpha}^{a} & q_{i}^{a} & Q_{I}^{a} & -d_{A}^{a} & m^{a}-d^{a} & d^{a}-m^{a}
\end{array}
$$

where

$$
m^{a}=\sum_{\alpha} m_{\alpha}^{a}, \quad d^{a}=\sum_{A} d_{A}^{a}
$$

The $n$ chiral multiplets $\Phi^{i}=\left(\phi^{i}, \psi^{i}\right)$ and their charges are determined by a presentation of $V$ as a symplectic $\mathrm{U}(1)^{R}$ quotient. The model has Fayet-Iliopoulos D-terms whose values $r^{a}$ correspond to the shift in the moment map for the $\mathrm{U}(1)^{R}$ action. The moduli space of classical vacua of a theory containing only these fields will be $V$ when the $r^{a}$ lie in a cone $\mathcal{K}_{V}$, the Kähler cone of $V$.

The $N$ Fermi multiplets $\Gamma^{I}$, with lowest components the left-moving fermions $\gamma^{I}$, satisfy a chirality condition ${ }^{3}$

$$
\overline{\mathcal{D}} \Gamma^{I}=\sqrt{2} E^{I}(\Sigma, \Phi), \quad E^{I}(\Sigma, \Phi)=\Sigma_{\mu} E^{I \mu}(\Phi),
$$

and their charges determine the bundle $\mathcal{E} \rightarrow V$ by the short exact sequence (SES)

$$
0 \longrightarrow \oplus_{\mu} \mathcal{O} \stackrel{E^{I \mu}}{\longrightarrow} \oplus_{I} \mathcal{O}\left(Q_{I}\right) \longrightarrow \mathcal{E} \longrightarrow 0
$$

This collection of fields with these couplings comprises what we refer to as the $V$ model [12]. It is not a conformal field theory, and will typically exhibit trivial IR behavior. The space

\footnotetext{
${ }^{2}$ We recall that a variety $V$ is Fano if and only if the anticanonical bundle $K_{V}$ of $V$ is ample.

${ }^{3}$ Unless otherwise specified, we use Einstein's summation convention throughout the paper.
} 
of $V$ models is parameterized by $r^{a}$ complexified by $\theta$ angles as well as the coefficients of the maps $E^{I \mu}$.

In general, there is a larger cone, which we call the geometric cone $\mathcal{K}_{c}$, in which the space of vacua has this character. More precisely, this is the cone in which the $V$ model as defined above has supersymmetric classical vacua. It is generated by $q_{i}$, and it is divided into phases. These correspond to subcones of $\mathcal{K}_{c}$, one of which is $\mathcal{K}_{V}$, separated by hyperplanes associated to $\mathrm{U}(1)$ subgroups of the gauge group which are unbroken at large $\phi$.

To construct our superconformal theory we augment the $V$ model by the $k$ chiral multiplets $P^{\alpha}=\left(p^{\alpha}, \chi^{\alpha}\right)$. For $r \in \mathcal{K}_{V}$ the space of classical vacua for the theory including these is the total space $V^{+}=\operatorname{tot}\left(\oplus_{\alpha} \mathcal{O}\left(-m_{\alpha}\right) \rightarrow V\right)$. We introduce as well $L$ Fermi multiplets $\Lambda^{A}$, whose lowest components are the left-moving fermions $\eta^{A}$, satisfying a chirality condition

$$
\overline{\mathcal{D}} \Lambda^{A}=\sqrt{2} E^{A}(P, \Sigma, \Phi), \quad \quad E^{A}(P, \Sigma, \Phi)=\Sigma_{\mu} P^{\alpha} E_{\alpha}^{A \mu}(\Phi) .
$$

The model with these fields and couplings will be referred to as the $V^{+}$model. Like the $V$ model, it will not in general be conformal. In addition to the parameters listed above, it is specified by the coefficients of the maps $E_{\alpha}^{A \mu}$.

The conformal model in which we are interested - the M model — is obtained from the $V^{+}$model by adding a superpotential interaction

$$
\left.\int d \theta^{+}\left(\Lambda^{A} H_{A}(\Phi)+\Gamma^{I} J_{I}(P, \Phi)\right)\right|_{\bar{\theta}^{+}=0}+\text { h.c. }
$$

where

$$
J_{I}(P, \Phi)=P^{\alpha} J_{I \alpha}(\Phi)
$$

subject to the conditions

$$
\sum_{A} H_{A} E_{\alpha}^{A \mu}+\sum_{I} J_{I \alpha} E^{\mu I}=0 \quad \forall \alpha, \mu
$$

required in order to preserve $(0,2)$ supersymmetry. For $r \in \mathcal{K}_{V}$ and generic $H_{A}$, the space of classical vacua is the complete intersection $M=\left\{\phi \in V \mid H_{A}(\phi)=0\right\}$. When this is nonsingular the $\Lambda$ fermions all acquire a mass and the light left-moving fermions take values in the bundle $\mathcal{V} \rightarrow M$ defined by the restriction to $M$ of the complex

$$
0 \longrightarrow \oplus_{\mu} \mathcal{O} \stackrel{E^{I \mu}}{\longrightarrow} \oplus_{I} \mathcal{O}\left(Q_{I}\right) \stackrel{J_{I \alpha}}{\longrightarrow} \oplus_{\alpha} \mathcal{O}\left(m_{\alpha}\right) \longrightarrow 0
$$

as $\mathcal{V}=\operatorname{Ker} J / \operatorname{Im} E$. We assume that $E$ is everywhere injective and $J$ everywhere surjective on $M$, and that $\mathcal{V}$ is a nonsingular, stable holomorphic vector bundle. These are the geometric models to which our methods apply. An intermediate step in the vanishing argument below involves setting $H=J=E=0$ in the M model — we refer to this as the $O$ model.

\footnotetext{
${ }^{4}$ These are in general subject to identifications, so this is an overparameterization.
} 
The chiral superfield $S$ and the chiral Fermi multiplet $\Xi$ are the "spectator" fields introduced in [13] to maintain the Kähler parameters $r^{a}$ as RG invariant quantities. In fact, the counterterm by which $r$ gets renormalized at one-loop is proportional to the sum of the gauge charges of the scalar fields in the theory. In our models this is in general nonzero (this is related to the fact that $V^{+}$is not Calabi-Yau). Introducing $S$ as above cancels this. The spectators earn their name because they interact via a superpotential

$$
\left.\int d \theta^{+} \Xi S\right|_{\bar{\theta}^{+}=0}+\text { h.c. . }
$$

This means these are massive fields and have no effect on the IR dynamics of the theory, so one might question the relevance of including them here. As we will see, in some cases accounting for their presence allows the argument to proceed where it might otherwise fail. This is, of course, a technical matter. If there are no instanton corrections in the presence of spectators there are none in their absence. This demonstrates an important caveat to our work, mentioned above. When the argument of [1] fails, we cannot assert that instanton corrections do destabilize the model, only that this particular argument that they do not is not valid.

Symmetries. The action (A.9)-(A.14) we have described is invariant under (0,2) SUSY and the gauge symmetry $\mathrm{U}(1)^{R}$, as well as a global $\mathrm{U}(1)_{R} \times \mathrm{U}(1)_{L}$ symmetry acting as

$$
\begin{array}{ccccccccc}
\text { fields } & P^{\alpha} & \Phi^{i} & \Gamma^{I} & \Lambda^{A} & S & \Xi & \Sigma_{\mu} & \Upsilon_{a} \\
\mathrm{U}(1)_{L} & 1 & 0 & -1 & 0 & 1 & -1 & -1 & 0 \\
\mathrm{U}(1)_{R} & 1 & 0 & 0 & 1 & 1 & 0 & 1 & 1
\end{array}
$$

While $\mathrm{U}(1)_{R}$ is believed to be the R-symmetry of the SCFT to which our model is supposed to flow, the global $\mathrm{U}(1)_{L}$ symmetry is equally important for our purposes: in heterotic compactifications, it can be used to construct a left-moving spectral flow operator and it provides a linearly realized component of the space-time group. The action of global symmetries on charged fields is of course defined up to an arbitrary action of the gauge symmetry generators. We have here chosen a representative action that is manifestly unbroken in the classical vacua (when $r \in \mathcal{K}_{V}$ ) comprising $M$.

These symmetries are respected by the classical action, but are in general anomalous in the presence of non-trivial gauge fields. The anomalies vanish when the charges satisfy

$$
\begin{aligned}
d^{a} & =\sum_{i} q_{i}^{a}, \\
m^{a} & =\sum_{I} Q_{I}^{a}, \\
\sum_{\alpha} m_{\alpha}^{a} m_{\alpha}^{b}+\sum_{i} q_{i}^{a} q_{i}^{b} & =\sum_{A} d_{A}^{a} d_{A}^{b}+\sum_{I} Q_{I}^{a} Q_{I}^{b} .
\end{aligned}
$$

In terms of our geometric data, the first two conditions reflect the fact that

$$
c_{1}\left(T_{M}\right)=c_{1}(\mathcal{V})=0,
$$


while the quadratic condition implies

$$
\operatorname{ch}_{2}\left(T_{M}\right)=\operatorname{ch}_{2}(\mathcal{V})
$$

Under these conditions, the $M$ model is believed to flow at low energies to a nontrivial superconformal field theory which is in the same moduli space as the nonlinear sigma model determined by the pair $(M, \mathcal{V})$. Nonperturbative effects (worldsheet instantons) which can destroy conformal invariance are captured by GLSM gauge instantons, which are the subject of our investigation here.

\section{The argument}

Let us first review the argument prescribed in [1] for the vanishing of the instanton contributions to the superpotential $W$ for space-time gauge singlets in a $(0,2)$ linear sigma model.

The goal is to probe for a background space-time superpotential $W$. A simple and direct way to achieve this is to compute the correlator $\mathcal{C}_{a b c}=\left\langle R_{a} R_{b} R_{c}\right\rangle$, where $R_{a}$ is the vertex operator representative for the Kähler modulus $\mathcal{R}_{a}$ of $V$. In fact, for each instanton the exponential factor $e^{I_{0}}$, where $I_{0}$ is the instanton classical action, contains all the dependence on $\mathcal{R}_{a}[2,14]$. The correlator $\mathcal{C}_{a b c}$ computes the third derivatives of $W$ with respect to $\mathcal{R}_{a}$, thus it determines $W$ up to quadratic terms in the $\mathcal{R}_{a}$. These terms are forbidden by standard $\alpha^{\prime}$ non-renormalization theorems, hence $\mathcal{C}_{a b c}$ determines $W$ directly.

The computation is most easily done in the half-twisted model (see appendix B). In this model, the supercharge $\overline{\mathcal{Q}}_{+}$becomes a nilpotent scalar symmetry generator, and correlators of $\overline{\mathcal{Q}}_{+}$-closed operators can be computed in its cohomology. On a genus-zero worldsheet, the twist can be realized by spectral flow insertions and calculations in the twisted model produce suitable correlators of the untwisted (physical) model.

In order to determine the linear model representative of the space-time mode $R_{a}$ we restrict our attention to the $(0,2)$ gauge multiplets. In fact, $R_{a}$ appears in the linear model through a F-I term. Moreover, gauge singlets must have $q_{L}=0$ and bosonic vertex operators have $q_{R}=1$. Finally,

$$
\overline{\mathcal{Q}}_{+} \lambda_{a,-}=0, \quad \mathcal{Q}_{+} \lambda_{a,-}=\frac{1}{2}\left(D_{a}-i f_{a, 01}\right)
$$

determine $R_{a}=\lambda_{a,-}$.

The first step of the argument is to show that $\mathcal{C}_{a b c}$ vanishes in the $O$ model. The idea is that the theory without superpotential has a very large symmetry, $G=\mathrm{U}(1)^{\oplus(n+N+k+L+2)}$, where each matter superfield is rotated separately, and the vertex operators $R_{a}$ are invariant under this. This symmetry is generically broken by superpotential couplings down to $U(1)_{L}$. If the zero-mode path integral measure ${ }^{5}$ in nontrivial topological sectors turns out not to be invariant under $G$, i.e. the symmetry is anomalous, then contributions to the invariant correlator $\mathcal{C}_{a b c}$ from this sector will vanish. In practice, we follow [1] and construct a

\footnotetext{
${ }^{5}$ We recall that the path integral for a correlator of $\overline{\mathcal{Q}}_{+}$-invariant operators localizes on fixed loci of $\overline{\mathcal{Q}}_{+}$, given by zero-modes.
} 
$\mathrm{U}(1)$ subgroup of $G$ that is rendered anomalous in all topological sectors by the twisting procedure, demonstrating that $\mathcal{C}_{a b c}=0$ identically in the $O$ model.

The second step uses the fact that $\mathcal{C}_{a b c}$ depends holomorphically on $J, H$ and $E$. One can then examine the contribution at arbitrary order in an expansion in these couplings. If there is no term that can possibly absorb the fermion zero-modes in the anomalous measure, then the correlator vanishes identically. This computation can be performed in each topological sector of the path integral.

In the untwisted model, the limiting point $J=H=E=0$ is of course highly singular. Both $\sigma$ and $p$ acquire zero-modes and the space of classical vacua is non-compact. Such a singularity can invalidate the order-by-order calculation described above. The key observation of [1] is that in suitable examples these dangerous zero-modes are absent in the half-twisted model. For example, the bosons $\sigma$ always have zero-modes, but in the twisted model (see appendix B) these fields acquire a spin and their zero-modes are absent. In general, as we shall see below, $p$ zero-modes are not completely removed by the twisting.

Another approach was presented in [1], where the vanishing of $\mathcal{C}_{a b c}$ at any instanton number follows from an appropriate counting of fermi zero-modes in the half-twisted model. This was applied in detail for heterotic compactifications described by half-linear sigma models, but it also extends to linear models as well. However, as pointed out above, the same assumption of compactness is required for this argument to be valid. For definiteness, we present our analysis of the linear model following the approach of [1] reviewed above, as our results will not depend on this choice.

\subsection{The quintic}

Let us review how all of this works for the linear model describing the deformations of the tangent bundle $T_{M}$ over the quintic hypersurface $M$ in $V=\mathbb{P}^{4}$. The gauge charges for the $(2,2)$ multiplets $\Phi^{i}=\left(\Phi^{i}, \Gamma^{i}\right)$ and $P=(P, \Lambda)$ are

$$
\begin{array}{ccccccc}
\text { fields } & P & \Phi^{1} & \Phi^{2} & \Phi^{3} & \Phi^{4} & \Phi^{5} \\
\mathrm{U}(1) & -5 & 1 & 1 & 1 & 1 & 1
\end{array}
$$

The Kähler cone $\mathcal{K}_{V}=\mathcal{K}_{c}$ here is simply given by $r \geq 0$ and the relevant instantons are defined by $\mathcal{K}_{V}^{\vee}=\{n \geq 0\}$. The $O$ model has as a target space the total space of the anticanonical bundle on $V$, tot $\left(\mathcal{O}(-5) \rightarrow \mathbb{P}^{4}\right)$. First of all, we check that the moduli space of gauge instantons for this model is compact. Indeed, we verify that there are no holomorphic sections of

$$
p \quad \leftrightarrow \quad \Gamma\left(K^{\frac{1}{2}} \otimes \mathcal{O}(-5 n)\right)
$$

and thus $p$ has no zero-modes. This, together with the fact that there are no zero-modes of $\sigma$ shows that the space of zero-modes is compact in any topological sector. Next, by looking at the degree of the line bundles of the half-twisted model in (B.7) we see that the 
relevant fermions zero-modes are

$\begin{array}{ccccc}\text { fields } & \bar{\psi}^{i} & \gamma^{i} & \bar{\eta} & \chi \\ \text { bundle } & \overline{\mathcal{O}}(n) & \mathcal{O}(n-1) & \mathcal{O}(5 n) & \overline{\mathcal{O}}(5 n-1) \\ \text { \# z.m. } & n+1 & n & 5 n+1 & 5 n\end{array}$

The fermion contribution to the zero-mode path-integral measure is then given by

$$
d \mu_{F}=d \bar{\lambda}_{-} d \bar{\eta} d \chi \prod_{i} d \bar{\psi}^{i} d \gamma^{i}
$$

Now, the $O$ model is invariant under a symmetry $\mathrm{U}(1)_{C}$ which assigns charge +1 to the multiplets $\Phi^{i}$ and leaves everything else invariant. Under this symmetry the measure above transforms with charge +5 . Hence, the correlator $\mathcal{C}$ vanishes in the $O$ model. The holomorphic superpotential couplings are given by

$$
\left.\mathcal{L}_{\text {Yuk }}\right|_{\bar{J}=\bar{H}=\bar{E}=0}=-\bar{\gamma}^{i} E^{i} \lambda_{+}+\gamma^{i} J_{i} \chi+\eta H_{, j} \psi^{j},
$$

where $H$ is a quintic polynomial defining the hypersurface $M, J_{i}$ are generic quartic polynomials and $E^{i}$ are generic linear polynomials subject to (2.8). Clearly, each coupling transforms under $\mathrm{U}(1)_{C}$ with either charge +5 or is neutral. By the argument above the correlator $\mathcal{C}$ vanishes in the full theory and there are no instanton corrections to the space-time superpotential.

\section{$3.2 \quad$ A counter-example}

Let us consider a two-parameter model with the following charge assignments

$$
\begin{array}{ccccccccccc}
\text { fields } & \Phi^{1,2,3} & \Phi^{4,5} & \Phi^{6,7} & \Lambda^{1} & \Lambda^{2} & \Gamma^{1,2} & \Gamma^{3} & \Gamma^{4,5,6,7} & P^{1} & P^{2} \\
\mathrm{U}(1)_{1} & 1 & 1 & 0 & -3 & -2 & 2 & 1 & 0 & -4 & -1 \\
\mathrm{U}(1)_{2} & 1 & 0 & 1 & -3 & -2 & 0 & 1 & 1 & -2 & -3
\end{array}
$$

In the geometric phase it describes a complete intersection $M$ of degree $(3,3)$ and $(2,2)$ hypersurfaces in the toric variety $V$ defined by the charges

$$
\left(\begin{array}{lllllll}
1 & 1 & 1 & 1 & 1 & 0 & 0 \\
1 & 1 & 1 & 0 & 0 & 1 & 1
\end{array}\right) .
$$

It is useful to write the maps defining the superpotential more explicitly. For ease of notation, let us denote $x=\left\{\phi^{1,2,3}\right\}, y=\left\{\phi^{4,5}\right\}, z=\left\{\phi^{6,7}\right\}$, as well as $\Gamma^{(1)}=\left\{\Gamma^{1,2}\right\}, \Gamma^{(2)}=$ $\left\{\Gamma^{3}\right\}, \Gamma^{(3)}=\left\{\Gamma^{4,5,6,7}\right\}$, and a condensed notation in which, e.g. $x^{k}$ denotes a generic homogeneous polynomial of degree $k$ in $\phi^{1,2,3}$. With this notation the maps are given as

$$
\begin{aligned}
& J_{(1)}=p^{1}\left(x^{2}+x y z+y^{2} z^{2}\right), \\
& J_{(2)}=p^{1}\left(x y^{2}+y^{3} z\right)+p^{2} z^{2}, \\
& J_{(3)}=p^{1}\left(x y^{3}+y^{4} z\right)+p^{2}\left(x z+y z^{2}\right),
\end{aligned}
$$


while the equations defining the complete intersections are

$$
\begin{aligned}
& H_{1}=x^{3}+x^{2} y z+x y^{2} z^{2}+y^{3} z^{3}, \\
& H_{2}=x^{2}+x y z+y^{2} z^{2} .
\end{aligned}
$$

The complete intersection $M$ is realized in the cone $\mathcal{K}_{V}=\left\{r_{1}>0, r_{1}-r_{2}<0\right\}$, where the irrelevant ideal is $B=(x y)(z)$. Since the $z$ 's are not both allowed to vanish and the coefficients in the expressions above are generic, we have that

$$
\begin{gathered}
\underbrace{\left(x z+y z^{2}\right)}_{4 \text { of these }}=x^{3}+x^{2} y z+x y^{2} z^{2}+y^{3} z^{3}=x^{2}+x y z+y^{2} z^{2}=0, \\
\underbrace{\left(x^{2}+x y z+y^{2} z^{2}\right)}_{3 \text { of these }}=x y^{2}+y^{3} z=\underbrace{\left(x y^{3}+y^{4} z\right)}_{4 \text { of these }}=x^{3}+x^{2} y z+x y^{2} z^{2}+y^{3} z^{3}=0,
\end{gathered}
$$

have no solutions compatible with the ideal $B$. Thus $p^{1}=p^{2}=0$ and there are no flat directions in this phase.

Now, note that $-p^{2} \in \mathcal{K}_{V}$ but $-p^{1} \notin \mathcal{K}_{V}$. Therefore there are instantons contributing for this phase for which $p^{1}$ develops zero-modes in the $O$ model. We can see this explicitly. Gauge instantons in this model have instanton numbers $n_{a} \in \mathcal{K}_{V}^{\vee}$, i.e. $n_{2}>0$ and $n_{1}+n_{2}>$ 0 . From appendix $\mathrm{B}$ we see that the zero-modes of $p^{1}$ are in one to one correspondence with holomorphic section of the bundle

$$
p^{1} \leftrightarrow \quad \Gamma\left(K^{\frac{1}{2}} \otimes \mathcal{O}\left(-4 n_{1}-2 n_{2}\right)\right),
$$

and the number of such sections is non-zero when $2 n_{1}+n_{2}<0$. The subcone defined by $\left(2 n_{1}+n_{2}<0\right) \cap \mathcal{K}_{V}^{\vee}$ is non empty, and the moduli space of gauge instantons of the $O$ model is not compact. The twisted $O$ model calculation in these sectors is ill-defined and the argument from holomorphy does not exclude instanton corrections to $\mathcal{C}_{a b c}$.

\section{The vanishing theorem}

The example of the previous section shows that a generic $(0,2)$ GLSM is not protected from worldsheet instanton corrections. In this section we undertake the task of constructing a class of models for which the vanishing theorem holds. In fact, a necessary condition for the vanishing argument to apply is that there exists a cone $\mathcal{K}_{V} \subseteq \mathcal{K}_{c}$ such that

1. the $\mathrm{M}$ model defined in $\mathcal{K}_{V}$ is nonsingular;

2. the $O$ model of the half-twisted theory has a compact moduli space of gauge instantons for any $n_{a} \in \mathcal{K}_{V}^{\vee}$.

Notice that as advertised above, due to the twist the bosons $\sigma$ acquire a spin and do not have zero-modes. A quick inspection at the form of the $E$-couplings (2.3) and (2.5) implies that setting $E=0$ does not lead to any singularities in the half-twisted theory. 


\section{1 $O$ model gauge instanton moduli space}

While the moduli space of gauge instantons for the $V$ model is compact, as we have seen above, there can be unbounded zero-modes coming from the $p^{\alpha}$ fields. This occurs when, for a given subcone $\mathcal{K}_{V} \subseteq \mathcal{K}_{c}$ we have $m_{\alpha} \notin \mathcal{K}_{V}$ for some $\alpha$. Hence, a necessary condition for the argument to work is that there exists a nonsingular subcone $\mathcal{K}_{V} \subseteq \mathcal{K}_{c}$ such that $m_{\alpha} \in \mathcal{K}_{V} \forall \alpha$.

The discussion so far did not take into account the spectator boson $s$, whose expectation value is set to zero, and whose zero-modes could also be fatal for our assumption of compactness. In order to establish when this is the case, we need the following simple fact: the cone $\widehat{\mathcal{K}}_{c}$ defined by adjoining the vector $m-d$ to $\mathcal{K}_{c}$ is convex unless $d-m \in \mathcal{K}_{c}$. In fact, $\widehat{\mathcal{K}}_{c}$ fails to be convex if we can write

$$
\sum_{i} \alpha_{i} q_{i}+\beta(m-d)=0,
$$

with $\alpha_{i}, \beta \geq 0$ and not all vanishing. Because $\mathcal{K}_{c}$ is convex by assumption, we must have $\beta$ strictly positive. This means $\beta(d-m)=\sum_{i} \alpha_{i} q_{i}$, i.e. $(d-m) \in \mathcal{K}_{c}$.

This little result suggests there are three separate cases we should consider:

1. $d-m \in \mathcal{K}_{V}$. By (B.7) $s$ has no zero-modes. In fact, by looking at the degree

$$
d_{S}=\left(m^{a}-d^{a}\right) n_{a},
$$

we have $d_{S} \leq 0 \forall n \in \mathcal{K}_{V}^{\vee}$.

2. $d-m \in \mathcal{K}_{c}$ but $d-m \notin \mathcal{K}_{V}$. In this case there exist $n \in \mathcal{K}_{V}^{\vee}$ such that $d_{S}>0$ and $s$ has zero-modes. The half-twisted $O$ model develops $s$-flat directions and is therefore singular.

3. $d-m \notin \mathcal{K}_{c}$. In this case $s$ always has zero-modes, but by the result above, together with the fact that a toric variety is compact if and only if the geometric cone is strongly convex, the moduli space of instantons for the $O$ model is nevertheless compact.

We can now summarize the set of conditions we are going to assume for our vanishing theorem: there exists a nonsingular subcone $\mathcal{K}_{V} \subseteq \mathcal{K}_{c}$ such that the gauge charge vectors for the fields $p^{\alpha}$ and $s$ satisfy

$$
m_{\alpha} \in \mathcal{K}_{V} \quad \forall \alpha, \quad d-m \in \mathcal{K}_{V} \quad \text { or } \quad d-m \notin \mathcal{K}_{c} .
$$

\subsection{A classical symmetry}

For the remaining of this section we restrict our attention to models obeying the conditions above. To proceed with the argument we need to construct a suitable $\mathrm{U}(1)_{C}$ subgroup of the symmetry group of the $O$ model. Let us choose the charges for the matter fields under this "classical" symmetry as

$$
\begin{array}{ccccccc}
\text { fields } & P^{\alpha} & \Phi^{i} & \Gamma^{I} & \Lambda^{A} & S & \Xi \\
\mathrm{U}(1)_{C} & 0 & q_{i}^{C} & Q_{I}^{C} & 0 & q_{S}^{C} & Q_{\Xi}^{C}
\end{array}
$$


while the gauge fields are invariant. This symmetry will be non-anomalous (before twisting) if

$$
\sum_{i} q_{i}^{a} q_{i}^{C}+\left(m^{a}-d^{a}\right) q_{S}^{C}=\sum_{I} Q_{I}^{a} Q_{I}^{C}+\left(d^{a}-m^{a}\right) Q_{\Xi}^{C}
$$

for $a=1, \ldots, R$.

The measure. First, let us look at the zero-mode contribution to the path integral measure. In particular, we are going to focus only on the fermionic part of the measure. In fact, the form of the maps (B.7), together with our assumption of compactness yield an exact balance between holomorphic and anti-holomorphic bosonic zero-modes. It is convenient to write the fermionic measure as

$$
d \mu_{F}=d \mu_{G} d \mu_{M} d \mu_{S},
$$

where the three factors correspond to the measure for the gauge, matter and spectator fields respectively. From (B.6) it follows that the gauge measure is simply given by

$$
d \mu_{G}=\prod_{a} d \bar{\lambda}_{-, a} .
$$

For the matter fields we have

$$
\begin{array}{ccccc}
\text { fields } & \chi^{\alpha} & \bar{\chi}^{\alpha} & \psi^{i} & \bar{\psi}^{i} \\
\text { bundle } & \bar{K}^{\frac{1}{2}} \otimes \overline{\mathcal{O}}\left(-d_{\alpha}\right) & \bar{K}^{\frac{1}{2}} \otimes \overline{\mathcal{O}}\left(d_{\alpha}\right) & \bar{K} \otimes \overline{\mathcal{O}}\left(-d_{i}\right) & \overline{\mathcal{O}}\left(d_{i}\right) \\
\text { \# z.m. } & \max \left(0,-d_{\alpha}\right)=-d_{\alpha} & \max \left(0, d_{\alpha}\right)=0 & \max \left(0,-d_{i}-1\right) & \max \left(0, d_{i}+1\right)
\end{array}
$$

where we used the fact that $m_{\alpha} \in \mathcal{K}_{V}$ implies $d_{\alpha} \leq 0$, as well as

$$
\begin{array}{ccccc}
\text { fields } & \gamma^{I} & \bar{\gamma}^{I} & \eta^{A} & \bar{\eta}^{A} \\
\text { bundle } & K^{\frac{1}{2}} \otimes \mathcal{O}\left(D_{I}\right) & K^{\frac{1}{2}} \otimes \mathcal{O}\left(-D_{I}\right) & K \otimes \mathcal{O}\left(D_{A}\right) & \mathcal{O}\left(-D_{A}\right) \\
\text { \# z.m. } & \max \left(0, D_{I}\right) & \max \left(0,-D_{I}\right) & \max \left(0, D_{A}-1\right) & \max \left(0,-D_{A}+1\right)
\end{array}
$$

The matter measure then reads

$$
d \mu_{M}=\prod_{\alpha} d \chi^{\alpha} \prod_{i \mid d_{i} \geq 0} d \bar{\psi}^{i} \prod_{i \mid d_{i}<0} d \psi^{i} \prod_{I \mid D_{I} \geq 0} d \gamma^{I} \prod_{I \mid D_{I}<0} d \bar{\gamma}^{I} \prod_{A \mid D_{A}>0} d \eta^{A} \prod_{A \mid D_{A} \leq 0} d \bar{\eta}^{A},
$$

and it is easy to check that it is gauge-invariant.

Finally, for the spectators

$$
\begin{array}{ccccc}
\text { fields } & \xi_{+} & \bar{\xi}_{+} & \xi_{-} & \bar{\xi}_{-} \\
\text {bundle } & \bar{K}^{\frac{1}{2}} \otimes \overline{\mathcal{O}}\left(-d_{S}\right) & \bar{K}^{\frac{1}{2}} \otimes \overline{\mathcal{O}}\left(d_{S}\right) & K^{\frac{1}{2}} \otimes \mathcal{O}\left(-d_{S}\right) & K^{\frac{1}{2}} \otimes \mathcal{O}\left(d_{S}\right) \\
\text { \# z.m. } & \max \left(0,-d_{S}\right) & \max \left(0, d_{S}\right) & \max \left(0,-d_{S}\right) & \max \left(0, d_{S}\right)
\end{array}
$$


Here we need to distinguish two cases, according to whether $d-m \in \mathcal{K}_{V}$ or $d-m \notin \mathcal{K}_{c}$, and we obtain

$$
d \mu_{S}= \begin{cases}d \xi_{+} d \xi_{-} & \text {if } d_{S}<0, \\ d \bar{\xi}_{+} d \bar{\xi}_{-} & \text {if } d_{S}>0\end{cases}
$$

Of course, if $d_{S}=0$ we simply ignore this factor.

Now we can finally determine how the measure transforms under the symmetry $\mathrm{U}(1)_{C}$ defined above. The gauge measure is invariant, while for the matter factor we obtain

$$
q^{C}\left(d \mu_{M}\right)=\sum_{i \mid d_{i} \geq 0}\left(d_{i}+1\right) q_{i}^{C}+\sum_{i \mid d_{i}<0}\left(-d_{i}-1\right)\left(-q_{i}^{C}\right)+\sum_{I \mid D_{I} \geq 0} D_{I}\left(-Q_{I}^{C}\right)+\sum_{I \mid D_{I}<0}\left(-D_{I}\right) Q_{I}^{C} .
$$

Finally, for the spectator measure in both cases of (4.12) we get

$$
q^{C}\left(d \mu_{S}\right)=d_{S}\left(q_{S}^{C}+Q_{\Xi}^{C}\right),
$$

Let us observe at this point that a very simple solution to (4.5) is given by

$$
q_{i}^{C}=Q_{I}^{C}=q_{S}^{C}=1, \quad Q_{\Xi}^{C}=0,
$$

where it is easy to verify that the equality holds by (2.12). Plugging these values into the expressions above we find that the total fermionic zero-mode measure in the twisted model transforms with charge $q^{C}\left(d \mu_{F}\right)=n$, where we recall that $n$ is the number of one-dimensional cones of the fan $\Delta_{V}$ for the toric variety $V$, and in particular is strictly positive. Thus, the fermion zero-modes cause $\mathrm{U}(1)_{C}$ to be anomalous, and $\mathcal{C}_{a b c}$ vanishes in the $O$ model.

The superpotential couplings. Let us turn to the analysis of the superpotential couplings in the action. The relevant Yukawa couplings are

$$
\left.\mathcal{L}_{\text {Yuk }}\right|_{\bar{J}=\bar{H}=\bar{E}=0}=-\bar{\gamma}^{I} E_{, \mu}^{I} \lambda_{\mu,+}+\gamma^{I} J_{I \alpha} \chi^{\alpha}+\eta^{A} H_{A, j} \psi^{j},
$$

where we have set $\sigma_{\mu}=p^{\alpha}=0$, as they have no zero-modes. We immediately see that all couplings, when non-zero, have the following lower bounds on the charges

$$
\begin{array}{cccc}
\text { couplings } & \bar{\gamma}^{I} E_{, \mu}^{I} \lambda_{\mu,+} & \gamma^{I} J_{I \alpha} \chi^{\alpha} & \eta^{A} H_{A, j} \psi^{j} \\
\mathrm{U}(1)_{C} & \geq 0 & \geq 2 & \geq 1
\end{array}
$$

In particular, we note that these values are all non-negative and therefore it is not possible to absorb the zero-modes in excess in the measure by bringing down fermion terms from the action. The correlator $\mathcal{C}_{a b c}$ thus vanishes at all orders in the superpotential couplings, which concludes the proof that instantons do not contribute to the space-time superpotential in our class of models.

Note that we ignored the anti-holomorphic functions $\bar{J}, \bar{H}$ and $\bar{E}$ in (4.16). This is in fact legitimate since, as observed above, half-twisted correlators of $\overline{\mathcal{Q}}_{+}$-closed operators have a holomorphic dependence on $J, H$ and $E$. 


\section{Outlook}

In this work, we investigated the details of the elegant argument of [1] for the absence of instanton corrections to the space-time superpotential in heterotic compactifications based on $(0,2)$ GLSMs. We have not been able to extend the argument to the most general case.

The immediate question raised is: are some of these vacua in fact destabilized by instantons? One clear way to resolve this would be to produce an argument that holds in more generality. It is possible, however, that no such argument can be found and that in fact instanton corrections do arise. One way to detect such corrections would be an indirect approach, in which properties of the solution, such as the dimension of the space of massless gauge-neutral scalar fields, are compared at different limiting points in the moduli space. A more direct approach would be to compute the instanton contributions explicitly. Perhaps the GLSM can provide a framework within which these calculations, which have proved difficult in general, are tractable.

On the other hand, we have now an extensive class of $(0,2)$ models which are truly conformally invariant. These can be used to explore the moduli space of $(0,2)$ theories without a $(2,2)$ locus, extending recent work that has focused on deformations of $(2,2)$ models [15-17]. In particular, one could look for special loci, e.g. good hybrid models [18] or Landau-Ginzburg points and hope to learn something about the structure of the resulting theories. In particular, hybrid models could be a promising laboratory for explicit computations of worldsheet instantons, given the simpler structure of rational curves on the lower dimensional base instead than on a CY three-fold.

Recently it has been shown that other "bad" things can happen in $(0,2)$ models [19]. In particular, it is shown, in the context of Landau-Ginzburg models, that the common assumption that accidental IR symmetries do not spoil the correspondence between operators in the IR and the ones in the UV is not guaranteed in $(0,2)$ models. When this occurs, the structure of the conformal manifold is dramatically modified. There is a priori no reason that would prevent the same phenomenon from happening in a generic phase of a GLSM. For example, one could realize one of the "accidental" LG theories as a phase of a GLSM and study how this pathology is realized in the geometric phase. This could shed new light on the conditions for the data $(M, \mathcal{V})$ to lead to consistent heterotic backgrounds.

\section{A Linear model conventions}

\section{A.1 (0,2) superspace}

We work in $(0,2)$ superspace $^{6}$ with coordinate $x^{ \pm}, \theta^{+}, \bar{\theta}^{+}$. The supercharges are given by

$$
\mathcal{Q}_{+}=\frac{\partial}{\partial \theta^{+}}+i \bar{\theta}^{+} \nabla_{+}, \quad \quad \overline{\mathcal{Q}}_{+}=-\frac{\partial}{\partial \bar{\theta}^{+}}-i \theta^{+} \nabla_{+},
$$

where $\partial_{+}=\partial / \partial x^{+}$and $\nabla_{+}$is the covariant gauge derivative. We also have the superderivatives

$$
\mathcal{D}_{+}=\frac{\partial}{\partial \theta^{+}}-i \bar{\theta}^{+} \nabla_{+}, \quad \quad \overline{\mathcal{D}}_{+}=-\frac{\partial}{\partial \bar{\theta}^{+}}+i \theta^{+} \nabla_{+} .
$$

\footnotetext{
${ }^{6}$ More details may be found in [20].
} 
The non-trivial anti-commutation relations are

$$
\left\{\mathcal{Q}_{+}, \overline{\mathcal{Q}}_{+}\right\}=-2 i \nabla_{+}, \quad\left\{\mathcal{D}_{+}, \overline{\mathcal{D}}_{+}\right\}=2 i \nabla_{+}
$$

\section{A.2 Field content}

There are two types of multiplets in the $(0,2)$ models we consider in this work.

1. Gauge fields multiplets. We have

$$
\begin{aligned}
V_{a,-} & =v_{a,-}-2 i \theta^{+} \bar{\lambda}_{a,-}-2 i \bar{\theta}^{+} \lambda_{a,-}+2 \theta^{+} \bar{\theta}^{+} D_{a} \\
\Sigma_{\mu} & =\sigma_{\mu}+\sqrt{2} \theta^{+} \lambda_{\mu,+}-i \theta^{+} \bar{\theta}^{+} \partial_{+} \sigma_{\mu}
\end{aligned}
$$

where $a=1, \ldots, R$ and $\mu=1, \ldots, m$. The multiplets $\Sigma_{\mu}$ are neutral chiral multiplets which in $(2,2)$ theories $^{7}$ combine with the $(0,2)$ gauge multiplets into $(2,2)$ gauge multiplets. The twisted chiral gauge invariant field strength is defined as

$$
\begin{aligned}
\Upsilon_{a} & =\left[\overline{\mathcal{D}}_{+}, \nabla_{-}\right] \\
& =i \overline{\mathcal{D}}_{+} V_{a,-}+\theta^{+} \nabla_{-} v_{a,+} \\
& =-2 \lambda_{a,-}-i \theta^{+}\left(D_{a}-i f_{a, 01}\right)-i \theta^{+} \bar{\theta}^{+} \partial_{+} \lambda_{a,-} .
\end{aligned}
$$

2. Matter multiplets. Here we have bosonic chiral (anti-chiral) multiplets

$$
\begin{aligned}
P^{\alpha} & =p^{\alpha}+\sqrt{2} \theta^{+} \chi^{\alpha}-i \theta^{+} \bar{\theta}^{+} \nabla_{+} p^{\alpha}, & \bar{P}^{\alpha} & =\bar{p}^{\alpha}-\sqrt{2} \bar{\theta}^{+} \bar{\chi}^{\alpha}+i \theta^{+} \bar{\theta}^{+} \nabla_{+} \bar{p}^{\alpha}, \\
\Phi^{i} & =\phi^{i}+\sqrt{2} \theta^{+} \psi^{i}-i \theta^{+} \bar{\theta}^{+} \nabla_{+} \phi^{i}, & \bar{\Phi}^{i} & =\bar{\phi}^{i}-\sqrt{2} \bar{\theta}^{+} \bar{\psi}^{i}+i \theta^{+} \bar{\theta}^{+} \nabla_{+} \bar{\phi}^{i} \\
S & =s+\sqrt{2} \theta^{+} \xi_{+}-i \theta^{+} \bar{\theta}^{+} \nabla_{+} s, & \bar{S} & =\bar{s}-\sqrt{2} \bar{\theta}^{+} \bar{\xi}_{+}+i \theta^{+} \bar{\theta}^{+} \nabla_{+} \bar{s},
\end{aligned}
$$

where $\alpha=1, \ldots, k$ and $i=1, \ldots, n$. We also have fermionic matter multiplets, which we again divide into three groups

$$
\begin{aligned}
\Gamma^{I} & =\gamma^{I}-\sqrt{2} \theta^{+} G^{I}-i \theta^{+} \bar{\theta}^{+} \nabla_{+} \gamma^{I}-\sqrt{2} \bar{\theta}^{+} E^{I}(\Phi, \Sigma), \\
\Lambda^{A} & =\eta^{I}-\sqrt{2} \theta^{+} F^{A}-i \theta^{+} \bar{\theta}^{+} \nabla_{+} \eta^{A}-\sqrt{2} \bar{\theta}^{+} E^{A}(P, \Phi, \Sigma), \\
\Xi & =\xi_{-}-\sqrt{2} \theta^{+} K-i \theta^{+} \bar{\theta}^{+} \nabla_{+} \xi_{-},
\end{aligned}
$$

as well as their complex conjugate

$$
\begin{aligned}
\bar{\Gamma}^{I} & =\bar{\gamma}^{I}-\sqrt{2} \bar{\theta}^{+} \bar{G}^{I}+i \theta^{+} \bar{\theta}^{+} \nabla_{+} \bar{\gamma}^{I}-\sqrt{2} \theta^{+} \bar{E}^{I}(\bar{\Phi}, \bar{\Sigma}) \\
\bar{\Lambda}^{A} & =\bar{\eta}^{I}-\sqrt{2} \bar{\theta}^{+} \bar{F}^{A}+i \theta^{+} \bar{\theta}^{+} \nabla_{+} \bar{\eta}^{A}-\sqrt{2} \theta^{+} \bar{E}^{A}(\bar{P}, \bar{\Phi}, \bar{\Sigma}), \\
\Xi & =\bar{\xi}_{-}-\sqrt{2} \bar{\theta}^{+} \bar{K}+i \theta^{+} \bar{\theta}^{+} \nabla_{+} \bar{\xi}_{-} .
\end{aligned}
$$

Here the indices are $I=1 \ldots, N$ and $A=1, \ldots, L$.

\footnotetext{
${ }^{7}$ In $(2,2)$ theories we have $R=m$.
} 


\section{A.3 The action}

Let us list the various terms that appear in the action for the $(0,2)$ linear models we consider in this work. We have the kinetic term for the gauge fields ${ }^{8}$

$$
\mathcal{L}_{G, K}=\frac{1}{8 e^{2}} \int d^{2} \theta^{+} \operatorname{Tr} \bar{\Upsilon}_{a} \Upsilon_{a}=\frac{1}{2 e^{2}}\left[2 i \bar{\lambda}_{a,-} \partial_{+} \lambda_{a,-}+D_{a}^{2}+f_{a, 01}^{2}\right],
$$

as well as the kinetic term for the $\Sigma_{\mu}$ fields

$$
\mathcal{L}_{\Sigma, K}=\frac{i}{2 e^{2}} \int d^{2} \theta^{+} \Sigma_{\mu} \nabla_{-} \Sigma_{\mu}=\frac{1}{e^{2}}\left[\partial_{+} \bar{\sigma}_{\mu} \partial_{-} \sigma_{\mu}+i \bar{\lambda}_{\mu,+} \partial_{-} \lambda_{\mu,+}\right] .
$$

Then we have the kinetic terms for the various matter fields. These are given as

$$
\begin{aligned}
\mathcal{L}_{\Phi, K}=\frac{i}{2} \int d^{2} \theta^{+} \bar{\Phi}^{i} \nabla_{-} \Phi^{i}= & \frac{1}{2}\left(\nabla_{+} \bar{\phi}^{i} \nabla_{-} \phi^{i}+\nabla_{-} \bar{\phi}^{i} \nabla_{+} \phi^{i}\right)+i \bar{\psi}^{i} \nabla_{-} \psi^{i} \\
& +i \sqrt{2} q_{i}^{a}\left(\bar{\psi}^{i} \bar{\lambda}_{a,-} \phi^{i}-\bar{\phi}^{i} \lambda_{a,-} \psi^{i}\right)+q_{i}^{a} D_{a} \bar{\phi}^{i} \phi^{i}, \\
\mathcal{L}_{\Gamma, K}=\frac{1}{2} \int d^{2} \theta^{+} \bar{\Gamma}^{I} \Gamma^{I}= & i \bar{\gamma}^{I} \nabla_{+} \gamma^{I}+\bar{G}^{I} G^{I}-\bar{E}^{I} E^{I} \\
& -\bar{\gamma}^{I} E_{, j}^{I} \psi^{j}-\bar{\gamma}^{I} E_{, \mu}^{I} \lambda_{\mu,+}-\bar{E}_{, j}^{I} \bar{\psi}^{j} \gamma^{I}-\bar{E}_{, \mu}^{I} \bar{\lambda}_{\mu,+} \gamma^{I},
\end{aligned}
$$

and similarly

$$
\begin{aligned}
& \mathcal{L}_{P, K}=\frac{i}{2} \int d^{2} \theta^{+} \bar{P}^{\alpha} \nabla_{-} P^{\alpha}= \frac{1}{2}\left(\nabla_{+} \bar{p}^{\alpha} \nabla_{-} p^{\alpha}+\nabla_{-} \bar{p}^{\alpha} \nabla_{+} p^{\alpha}\right)+i \bar{\chi}^{\alpha} \nabla_{-} \chi^{\alpha} \\
&-i \sqrt{2} m_{\alpha}^{a}\left(\bar{\chi}^{\alpha} \bar{\lambda}_{a,-} p^{\alpha}-\bar{p}^{\alpha} \lambda_{a,-} \chi^{\alpha}\right)-m_{\alpha}^{a} D_{a} \bar{p}^{\alpha} p^{\alpha}, \\
& \mathcal{L}_{\Lambda, K}=\frac{1}{2} \int d^{2} \theta^{+} \bar{\Lambda}^{A} \Lambda^{A}= i \bar{\eta}^{A} \nabla_{+} \eta^{A}+\bar{F}^{A} F^{A}-\bar{E}^{A} E^{A} \\
&-\bar{\eta}^{A} E_{, j}^{A} \psi^{j}-\bar{\eta}^{A} E_{, \mu}^{A} \lambda_{\mu,+}-\bar{E}_{, j}^{A} \bar{\psi}^{j} \eta^{A}-\bar{E}_{, \mu}^{A} \bar{\lambda}_{\mu,+} \eta^{A}, \\
& \mathcal{L}_{S, K}=\frac{i}{2} \int d^{2} \theta^{+} \bar{S} \nabla_{-} S= \frac{1}{2}\left(\nabla_{+} \bar{s} \nabla_{-} s+\nabla_{-} \bar{s} \nabla_{+} s\right)+i \bar{\xi}_{+} \nabla_{-} \xi_{+} \\
&+i \sqrt{2}\left(m^{a}-d^{a}\right)\left(\bar{\xi}_{+} \bar{\lambda}_{a,-} s-\lambda_{a,-} \xi_{+} \bar{s}\right)+\left(m^{a}-d^{a}\right) D_{a} \bar{s} s, \\
& \mathcal{L}_{\Xi, K}=\frac{1}{2} \int d^{2} \theta^{+} \bar{\Xi} \Xi=i \bar{\xi}_{-} \nabla_{+} \xi_{-}+\bar{K} K .
\end{aligned}
$$

The Fayet-Iliopoulos terms action arises as a linear twisted superpotential for the twisted chiral fields $\Upsilon_{a}$

$$
\mathcal{L}_{\mathrm{F}-\mathrm{I}}=\left.\frac{1}{4} \int d \theta^{+} \Upsilon_{a} \tau^{a}\right|_{\bar{\theta}^{+}=0}+\text { h.c. }=-D_{a} r^{a}+\frac{\theta^{a}}{2 \pi} f_{a, 01}
$$

where $\tau^{a}=i r^{a}+\theta^{a} / 2 \pi$ are the complexified F-I parameters. Finally, the matter superpotential is a sum of three terms

$$
\begin{aligned}
& \mathcal{L}_{J}=-\left.\frac{1}{\sqrt{2}} \int d \theta^{+} \Gamma^{I} J_{I}(P, \Phi)\right|_{\bar{\theta}^{+}=0}+\text { h.c. }=G^{I} p^{\alpha} J_{I \alpha}+\gamma^{I} p^{\alpha} J_{I \alpha, j} \psi^{j}+\gamma^{I} J_{I \alpha} \chi^{\alpha}+\text { h.c. } \\
& \mathcal{L}_{H}=-\left.\frac{1}{\sqrt{2}} \int d \theta^{+} \Lambda^{A} H_{I}(\Phi)\right|_{\bar{\theta}^{+}=0}+\text { h.c. }=F^{A} H_{A}+\eta^{A} H_{A, j} \psi^{j}+\text { h.c. } \\
& \mathcal{L}_{S}=-\left.\frac{1}{\sqrt{2}} \int d \theta^{+} \Xi S\right|_{\bar{\theta}^{+}=0}+\text { h.c. }=K s+\xi_{-} \xi_{+}+\text {h.c. }
\end{aligned}
$$

\footnotetext{
${ }^{8}$ For simplicity, we have set equal all gauge coupling constants.
} 
The last term explicitly shows that all the excitations of the spectator fields are massive and they do not affect the low energy physics. In (A.14) we implemented the form for the superpotential (2.7).

\section{B The half-twist}

In order to probe for a background space-time superpotential $W$ it is convenient to halftwist the model, that is we twist by $J_{H}=J_{R} / 2$, where $J_{R}$ is the generator of the rightmoving R-symmetry. We implement this by redefining the Lorentz generator $J_{L}$ as

$$
J_{L}^{\prime}=J_{L}-J_{R} / 2
$$

Explicitly, for the gauge fields we have

$$
\begin{array}{ccccccc}
\text { fields } & \sigma_{\mu} & \bar{\sigma}_{\mu} & \lambda_{+, \mu} & \bar{\lambda}_{+, \mu} & \lambda_{-, a} & \bar{\lambda}_{-, a} \\
J_{L} & 0 & 0 & \frac{1}{2} & \frac{1}{2} & -\frac{1}{2} & -\frac{1}{2} \\
J_{L}^{\prime} & -\frac{1}{2} & \frac{1}{2} & \frac{1}{2} & \frac{1}{2} & -1 & 0
\end{array}
$$

while for the matter fields we have instead

$$
\begin{array}{ccccccccc}
\text { fields } & p^{\alpha} & \bar{p}^{\alpha} & \phi^{i} & \bar{\phi}^{i} & \chi^{\alpha} & \bar{\chi}^{\alpha} & \psi^{i} & \bar{\psi}^{i} \\
J_{L} & 0 & 0 & 0 & 0 & \frac{1}{2} & \frac{1}{2} & \frac{1}{2} & \frac{1}{2} \\
J_{L}^{\prime} & -\frac{1}{2} & \frac{1}{2} & 0 & 0 & \frac{1}{2} & \frac{1}{2} & 1 & 0
\end{array}
$$

and

$$
\begin{array}{ccccccccccc}
\text { fields } & \eta^{A} & \bar{\eta}^{A} & \gamma^{I} & \bar{\gamma}^{I} & s & \bar{s} & \xi_{+} & \bar{\xi}_{+} & \xi_{-} & \bar{\xi}_{-} \\
J_{L} & -\frac{1}{2} & -\frac{1}{2} & -\frac{1}{2} & -\frac{1}{2} & 0 & 0 & \frac{1}{2} & \frac{1}{2} & -\frac{1}{2} & -\frac{1}{2} \\
J_{L}^{\prime} & -1 & 0 & -\frac{1}{2} & -\frac{1}{2} & -\frac{1}{2} & \frac{1}{2} & \frac{1}{2} & \frac{1}{2} & -\frac{1}{2} & -\frac{1}{2}
\end{array}
$$

In the twisted model the supercharge $\overline{\mathcal{Q}}_{+}$becomes a worldsheet scalar. $\overline{\mathcal{Q}}_{+}$-exact operators will decouple from the correlators of $\overline{\mathcal{Q}}_{+}$-closed fields, to which we restrict our attention. In particular, the kinetic terms for all fields are $\overline{\mathcal{Q}}_{+}$-exact up to a topological term determined by the gauge bundle on the world-sheet $\Sigma=\mathbb{P}^{1}$ via the instanton numbers

$$
n_{a}=-\frac{1}{2 \pi} \int f_{a, 01} \text {. }
$$

The integral over field configurations breaks up into a sum over topological sectors indexed by $n_{a}$. For $r \in \mathcal{K}_{V}$, these lie in $\mathcal{K}_{V}^{\vee}$, and the classical action weights the contribution of each sector by $\prod_{a} q_{a}^{n_{a}}$ where $q_{a}=e^{-2 \pi r_{a}+i \theta_{a}}$. Extracting this topological contribution we can perform the computation within each topological sector semiclassically, and the path integral reduces to an integral over the zero modes of the fields. 
The space of zero modes to which the path integral reduces in each sector can be represented as the space of (anti-) holomorphic sections of appropriate line bundles over $\Sigma$. Explicitly, the gauge fields take values in

$$
\begin{aligned}
& \sigma_{a} \leftrightarrow K^{\frac{1}{2}} \quad \bar{\sigma}_{a} \leftrightarrow \bar{K}^{\frac{1}{2}} \\
& \lambda_{+, \mu} \leftrightarrow \bar{K}^{\frac{1}{2}} \quad \bar{\lambda}_{+, \mu} \leftrightarrow \bar{K}^{\frac{1}{2}} \\
& \lambda_{-, a} \leftrightarrow K \quad \bar{\lambda}_{-, a} \leftrightarrow \quad \leftrightarrow \quad \mathcal{O}
\end{aligned}
$$

where $K=\mathcal{O}(-2)$ is the canonical bundle. For the matter fields we have instead

$$
\begin{aligned}
& p^{\alpha} \quad \leftrightarrow \quad K^{\frac{1}{2}} \otimes \mathcal{O}\left(d_{\alpha}\right) \quad \bar{p}^{\alpha} \quad \leftrightarrow \quad \bar{K}^{\frac{1}{2}} \otimes \overline{\mathcal{O}}\left(d_{\alpha}\right) \\
& \phi^{i} \leftrightarrow \quad \mathcal{O}\left(d_{i}\right) \quad \phi^{i} \leftrightarrow \overline{\mathcal{O}}\left(d_{i}\right) \\
& \psi^{i} \quad \leftrightarrow \quad \bar{K} \otimes \overline{\mathcal{O}}\left(-d_{i}\right) \quad \bar{\psi}^{i} \leftrightarrow \quad \quad \overline{\mathcal{O}}\left(d_{i}\right) \\
& \gamma^{I} \leftrightarrow K^{\frac{1}{2}} \otimes \mathcal{O}\left(D_{I}\right) \quad \bar{\gamma}^{I} \quad \leftrightarrow \quad K^{\frac{1}{2}} \otimes \mathcal{O}\left(-D_{I}\right) \\
& \eta^{A} \leftrightarrow \quad K \otimes \mathcal{O}\left(D_{A}\right) \quad \bar{\eta}^{A} \quad \leftrightarrow \quad \mathcal{O}\left(-D_{A}\right) \\
& \chi^{\alpha} \leftrightarrow \quad \bar{K}^{\frac{1}{2}} \otimes \overline{\mathcal{O}}\left(-d_{\alpha}\right) \quad \bar{\chi}^{\alpha} \quad \leftrightarrow \quad \bar{K}^{\frac{1}{2}} \otimes \overline{\mathcal{O}}\left(d_{\alpha}\right) \\
& s \quad \leftrightarrow \quad K^{\frac{1}{2}} \otimes \mathcal{O}\left(d_{S}\right) \quad \bar{s} \quad \leftrightarrow \quad \bar{K}^{\frac{1}{2}} \otimes \overline{\mathcal{O}}\left(d_{S}\right) \\
& \xi_{+} \quad \leftrightarrow \quad \bar{K}^{\frac{1}{2}} \otimes \overline{\mathcal{O}}\left(-d_{S}\right) \quad \bar{\xi}_{+} \quad \leftrightarrow \quad \bar{K}^{\frac{1}{2}} \otimes \overline{\mathcal{O}}\left(d_{S}\right) \\
& \xi_{-} \leftrightarrow K^{\frac{1}{2}} \otimes \mathcal{O}\left(-d_{S}\right) \quad \bar{\xi}_{-} \leftrightarrow K^{\frac{1}{2}} \otimes \mathcal{O}\left(d_{S}\right)
\end{aligned}
$$

where the various degrees are defined as

$$
d_{\alpha}=-m_{\alpha}^{a} n_{a}, \quad d_{i}=q_{i}^{a} n_{a}, \quad D_{I}=Q_{I}^{a} n_{a}, \quad D_{A}=-d_{A}^{a} n_{a}, \quad d_{S}=\left(m^{a}-d^{a}\right) n_{a} .
$$

Note that it turned out to be convenient to use a hermitian metric on the appropriate bundles on $\mathbb{P}^{1}$ to redefine some of the fields [21]. By examining the half-twisted action it is possible to show that the couplings $\bar{\tau}_{a}$ as well as $\bar{H}, \bar{J}$ and $\bar{E}$ only appear in $\overline{\mathcal{Q}}_{+}$-exact terms. One very important consequence of this for us is that in the half-twisted theory, correlators of $\overline{\mathcal{Q}}_{+}$-closed operators are holomorphic in $J, H$ and $E$, thus for the purpose of our computations we can set $\bar{J}=\bar{H}=\bar{E}=0$.

Open Access. This article is distributed under the terms of the Creative Commons Attribution License (CC-BY 4.0), which permits any use, distribution and reproduction in any medium, provided the original author(s) and source are credited.

\section{References}

[1] C. Beasley and E. Witten, Residues and world sheet instantons, JHEP 10 (2003) 065 [hep-th/0304115] [INSPIRE].

[2] M. Dine, N. Seiberg, X.G. Wen and E. Witten, Nonperturbative effects on the string world sheet, Nucl. Phys. B 278 (1986) 769 [INSPIRE]. 
[3] L.J. Dixon, Some world sheet properties of superstring compactifications, on orbifolds and otherwise, lectures given at the 1987 ICTP Summer Workshop in High Energy Phsyics and Cosmology, June 29-August 7, Trieste, Italy (1987).

[4] J. Distler, Resurrecting (2,0) compactifications, Phys. Lett. B 188 (1987) 431 [InSPIRE].

[5] J. Distler and B.R. Greene, Aspects of $(2,0)$ string compactifications, Nucl. Phys. B 304 (1988) 1 [inSPIRE].

[6] P. Berglund, P. Candelas, X. de la Ossa, E. Derrick, J. Distler and T. Hubsch, On the instanton contributions to the masses and couplings of $E_{6}$ singlets, Nucl. Phys. B 454 (1995) 127 [hep-th/9505164] [INSPIRE].

[7] V. Braun, M. Kreuzer, B.A. Ovrut and E. Scheidegger, Worldsheet instantons and torsion curves, part A: direct computation, JHEP 10 (2007) 022 [hep-th/0703182] [INSPIRE].

[8] P.S. Aspinwall and M.R. Plesser, Elusive worldsheet instantons in heterotic string compactifications, Proc. Symp. Pure Math. 85 (2012) 33 [arXiv:1106.2998] [InSPIRE].

[9] E. Witten, Phases of $N=2$ theories in two-dimensions, Nucl. Phys. B 403 (1993) 159 [hep-th/9301042] [INSPIRE].

[10] E. Silverstein and E. Witten, Criteria for conformal invariance of $(0,2)$ models, Nucl. Phys. B 444 (1995) 161 [hep-th/9503212] [INSPIRE].

[11] A. Basu and S. Sethi, World sheet stability of $(0,2)$ linear $\sigma$-models, Phys. Rev. D 68 (2003) 025003 [hep-th/0303066] [INSPIRE].

[12] D.R. Morrison and M.R. Plesser, Summing the instantons: quantum cohomology and mirror symmetry in toric varieties, Nucl. Phys. B 440 (1995) 279 [hep-th/9412236] [INSPIRE].

[13] J. Distler, Notes on $(0,2)$ superconformal field theories, hep-th/9502012.

[14] M. Dine, N. Seiberg, X.G. Wen and E. Witten, Nonperturbative effects on the string world sheet. 2, Nucl. Phys. B 289 (1987) 319 [INSPIRE].

[15] J. McOrist and I.V. Melnikov, Summing the instantons in half-twisted linear $\sigma$-models, JHEP 02 (2009) 026 [arXiv:0810.0012] [InSPIRE].

[16] P.S. Aspinwall and B. Gaines, Rational curves and $(0,2)$-deformations, J. Geom. Phys. 88 (2014) 1 [arXiv:1404.7802] [InSPIRE].

[17] R. Donagi, Z. Lu and I.V. Melnikov, Global aspects of $(0,2)$ moduli space: toric varieties and tangent bundles, Commun. Math. Phys. 338 (2015) 1197 [arXiv:1409.4353] [INSPIRE].

[18] M. Bertolini, I.V. Melnikov and M.R. Plesser, Hybrid conformal field theories, JHEP 05 (2014) 043 [arXiv: 1307.7063] [INSPIRE].

[19] M. Bertolini, I.V. Melnikov and M.R. Plesser, Accidents in (0,2) Landau-Ginzburg theories, JHEP 12 (2014) 157 [arXiv: 1405.4266] [INSPIRE].

[20] J. Wess and J. Bagger, Supersymmetry and supergravity, Princeton University Press, Princeton U.S.A. (1992).

[21] S.H. Katz and E. Sharpe, Notes on certain $(0,2)$ correlation functions, Commun. Math. Phys. 262 (2006) 611 [hep-th/0406226] [INSPIRE]. 